\title{
Behavioral inhibition and behavioral activation in college students: The effects of aversive control
}

\author{
Chad S. Brice \\ West Virginia University
}

Follow this and additional works at: https://researchrepository.wvu.edu/etd

\section{Recommended Citation}

Brice, Chad S., "Behavioral inhibition and behavioral activation in college students: The effects of aversive control" (2009). Graduate Theses, Dissertations, and Problem Reports. 2901.

https://researchrepository.wvu.edu/etd/2901

This Thesis is protected by copyright and/or related rights. It has been brought to you by the The Research Repository @ WVU with permission from the rights-holder(s). You are free to use this Thesis in any way that is permitted by the copyright and related rights legislation that applies to your use. For other uses you must obtain permission from the rights-holder(s) directly, unless additional rights are indicated by a Creative Commons license in the record and/ or on the work itself. This Thesis has been accepted for inclusion in WVU Graduate Theses, Dissertations, and Problem Reports collection by an authorized administrator of The Research Repository @ WVU. For more information, please contact researchrepository@mail.wvu.edu. 
Behavioral Inhibition and Behavioral Activation in College Students: The effects of aversive control

\title{
Chad S. Brice
}

Thesis submitted to The Eberly College of Arts and Sciences at West Virginia University in partial fulfillment of the requirements for the degree of

\author{
Master of Science \\ in \\ Psychology
}

Tracy L. Morris, Ph.D., Chair Michael Perone, Ph.D.

Joseph R. Scotti, Ph.D.

Department of Psychology

Morgantown, West Virginia
2009

Keywords: Behavioral inhibition, Behavioral activation, Aversive control Copyright 2009 Chad S. Brice 


\title{
ABSTRACT \\ Behavioral Inhibition and Behavioral Activation in College Students: The effects of aversive control
}

\begin{abstract}
Chad Brice
Negative reinforcement is utilized in many contexts in our society but its differential effects in relation to individual differences are rarely studied. It is very similar in form to punishment in that they are both forms of aversive control, and only differs in the sense that negative reinforcement is a technique utilized to increase rather than reduce a behavior. Researchers, starting with Gray, have shown that individuals higher in inhibition and anxiety are more responsive to punishment, while individuals who are more impulsive are more sensitive to rewards. The positive relation between anxiety and sensitivity to punishment suggests that individuals higher in inhibition and anxiety might also be more responsive to negative reinforcement. Consistent findings have been established for how individuals who are more impulsive respond on tasks utilized to measure inhibition and approach, while results for anxious individuals are inconsistent. On go/no-go tasks specifically, impulsive individuals are less likely to inhibit responses that could result in loss than individuals who are less impulsive. No empirical explanations have been established for why individuals higher in anxiety do not respond in a consistent manner on these tasks. Gray's theory of behavioral inhibition would assume that individuals higher in anxiety would be more likely to inhibit responses and even avoid opportunities for reward that may result in aversive consequences. The current research aimed to study the relation between level of inhibition and anxiety, and how individuals respond to negative reinforcement using a computerized go/no-go task. Despite inconsistent findings on previous go/no-go tasks, individuals higher in anxiety might alter responding more consistently to an increase in negative reinforcement. The current study did not identify any individual differences in how individuals responded to negative reinforcement, but does offer a paradigm to further research in the area of negative reinforcement. This study outlines the methods used and offers suggestions for improving the methods in the future.
\end{abstract}




\section{ACKNOWLEDGEMENTS}

Thank you to Tracy Morris Ph.D. for fostering my interest in translational research and encouraging my exploration of experimental designs. Thank you to Dr. Perone and Dr. Scotti for shaping my theoretical orientation and understanding of behavioral principles. A special thanks to Anne Foreman for her

programming expertise and assistance with the Aversive Control Task. Finally, thank you to Chris Drescher for his commitment and responsibility throughout the data collection process. 
TABLE OF CONTENTS

TITLE PAGE

$\begin{array}{lll}\text { ABSTRACT } & \text { ii }\end{array}$

ACKNOWLEDGEMENTS $\quad$ iii

TABLE OF CONTENTS iv

LIST OF TABLES $\quad$ v

INTRODUCTION

GRAY'S THEORY OF BEHAVIORAL INHIBITION AND BEHAVIORAL APPROACH 1

GRAY'S THEORY AND ITS ASSOCIATION WITH PSYCHOLOGICAL DISORDERS 3

DISTINCTION BETWEEN ANXIETY AND SENSITIVITY TO PUNISHMENT 4

ANXIETY AND PERFORMANCE

$\begin{array}{ll}\text { SCHOOL PERFORMANCE AND ANXIETY } & 7\end{array}$

$\begin{array}{ll}\text { AVERSIVE CONTROL } & 7\end{array}$

ALTERNATIVE PERFORMANCE TASKS $\quad 8$

$\begin{array}{ll}\text { HYPOTHESES } & 9\end{array}$

$\begin{array}{ll}\text { METHOD } & 10\end{array}$

$\begin{array}{ll}\text { PARTICIPANTS } & 10\end{array}$

$\begin{array}{lr}\text { MEASURES } & 10\end{array}$

$\begin{array}{ll}\text { PROCEDURE } & 13\end{array}$

$\begin{array}{ll}\text { RESULTS } & 14\end{array}$

$\begin{array}{ll}\text { DISCUSSION } & 17\end{array}$

REFERENCES 22

$\begin{array}{ll}\text { TABLES } & 25\end{array}$

$\begin{array}{ll}\text { APPENDICES } & 30\end{array}$

$\begin{array}{ll}\text { CURRICULUM VITA } & 34\end{array}$ 
LIST OF TABLES

TABLE 1: DESCRIPTIVE STATISTICS FOR ASSESSMENT MEASURES

TABLE 2: DESCRIPTIVE STATISTICS BY ACT STIMULI

TABLE 3: CORRELATIONS AMONG PRIMARY STUDY VARIABLES

TABLE 4: LINEAR REGRESSSIONS FOR PERFORMANCE MEASURES BY SENSITIVITY TO PUNISHMENT AND BAI SCORES

TABLE 5: LINEAR REGRESSIONS FOR PERFORMANCE MEASURES BY SENSITIVITY TO 29 REWARD AND BIS-11 SCORES 
Behavioral Inhibition 1

Behavioral Inhibition and Behavioral Activation in College Students:

The effects of aversive control

Anxiety disorders have been associated with performance deficits including academic and social functioning, but the underlying mechanisms that influence these relations have not been extensively researched with a laboratory model (Langley, 2004; Wood 2006). Two key features of anxiety are the negative reinforced behaviors of avoidance and escape. Avoidance is characterized by an organism engaging in behaviors to remove or get away from stimuli associated with negative consequences. Escape is characterized by behaviors that serve to remove or get away from aversive events that the organism is already experiencing. It is unclear whether performance deficits in individuals with anxiety disorders are related to avoidance and escape learning or distracting physiological arousal associated with the performance task. Gray (1970) proposed that individuals higher in anxiety learn more readily from punishment than individuals lower in anxiety. Punishment in this case refers to the reduction of behaviors that result in negative consequences. Avoidance and escape maintained behaviors are difficult to punish because they are reinforced every time the organism engages in the behavior without negative consequences. One way to punish these behaviors is negative reinforcement for behaviors that are incompatible with previously learned escape and avoidance behaviors. Though not stated directly, Gray's theory supports the notion that increasing negative reinforcement should improve performance in individuals with anxiety disorders, by punishing avoidance in situations in which approach behaviors would be rewarded. The goal of this study is to develop a laboratory model to test the impact of aversive negative reinforcement on individuals who have higher levels of anxiety symptoms.

\section{Gray's Theory of Behavioral Inhibition and Behavioral Approach}

Gray's theory of brain function and behavior initially proposed that there are two major motivational systems that govern behavior and affect. Those two systems are the behavioral inhibition system (BIS) and the behavioral approach system (BAS; Gray 1970, 1978). The BIS is related to high avoidance and low impulsivity due to sensitivity to punishment or lack of reward associated with conditioned stimuli. Conversely, the BAS is related to sensitivity to reward and is marked by approach 
Behavioral Inhibition 2 behaviors associated with conditioned stimuli. In other words, individuals with stronger BAS functioning are more likely to engage in behaviors that they have been rewarded for in the past than individuals with weaker BAS functioning.

The BIS and BAS systems are theorized to be located in different regions of the brain and are proposed to operate orthogonal to each other. Gray (1982) determined by examining the brains of rats that the BIS is located in the septo-hippocampal region of the brain. He administered anxiolytics to the rats and found that these drugs led to less inhibition of lever-pressing followed by a shock and less activity in the septo-hippocampal region of the brain. The BAS is represented more accurately by heart rate activity than somatic activity (Fowles, 1980). The orthogonal nature of these systems indicates that an individual can have any combination of levels of functioning between the two systems.

In support of the BIS and it's relation to anxiety disorders, Corr, Pickering, and Gray (1997) found that individuals with an anxiety disorder diagnosis were more likely to improve their performance on a procedural learning task under a condition of punishment than individuals under a control condition. Corr et. al. (1997) stated that the procedural learning task was chosen because it was associated with phylogenetically older brain systems located in the hindbrain. This type of learning made the generalizability between species from Gray's initial study with rats to humans more likely. It should be noted that procedural learning is associated with the cerebellum and can be biologically differentiated from declarative learning, primarily formed by the hippocampus. The ability of individuals with anxiety disorders to learn from punishment should not be generalized to all types of learning based on this research alone. Higher level learning may involve more systems and variables than simply the BIS and the BAS.

Gray later proposed that, in addition to the BIS and BAS, there was a third motivational system that governs behavior and affect. This third system, the fight/flight system, is associated with arousal due to unconditioned pain and non-reward (Gray 1982, 1987). The fight/flight system is associated with activity in the central grey, medial hypothalamus, and the amygdala. This system accounts for escape from unconditioned stimuli and defensive aggression. This system could explain performance errors and 
Behavioral Inhibition 3

avoidance associated with novel situations. The arousal associated with this system could also explain the negative affectivity, Zinbarg and Barlow (1996) describe as the central defining feature of anxiety. It is not surprising that individuals experiencing anxious arousal would report feelings of discomfort or dysphoria and subsequently engage in escape from those feelings.

\section{Gray’s Theory and its Association with Psychological Disorders}

Researchers have used Gray's theory to explain anxiety, psychopathy, depression, conduct disorder, and ADHD by showing the relation of these disorders to sensitivity to punishment and reward (Gray, 1982; Newman, MacCoon, Vaughn, \& Sadeh, 2005; Harmon-Jones \& Allen, 1997; Carver \& White, 1994; Quay, 1997; Shapiro, Quay, Hogan, \& Schwartz, 1998). According to Gray, sensitivity to punishment and reward are relative strengths and weaknesses of the BIS and BAS systems, respectively. Gray's theory proposes that anxiety is related to increased BIS activity independent of BAS activity.

Conversely, psychopathy is associated with a strong BAS, but has been identified in individuals with both strong and weak BIS (Newman et al.., 2005). Newman describes two types of psychopathy, primary and secondary. Primary psychopathy is associated with a biological predisposition, but secondary psychopathy is associated with environmental contributions, such as low IQ, social disadvantage or some other psychopathology. Both types of psychopathy are indicative of a strong BAS, but primary psychopathy is associated with low BIS functioning, while secondary psychopathy has varying levels of BIS functioning, further supporting the independent functioning of the two systems. Similar to psychopathy, individuals with conduct disorder display strong sensitivity to reward as demonstrated by perseveration on a card-playing task (Shapiro et al., 1988).

Some disorders are explained by deficits in BIS and BAS functioning instead of increased activity. Harmon-Jones (1997) found that individuals with low EEG activity in the frontal cortex, a symptom typical in depression, had a strong negative relation with self-reported BAS functioning using the BIS/BAS scales (Carver \& White, 1994). ADHD is explained by low BIS functioning, which is consistent with the amount of impulsivity associated with the disorder (Quay, 1997). 
Behavioral Inhibition 4

Despite these findings associated with the BIS and BAS, Gray's theory does not explain

comorbidity with disorders that seem to have conflicting levels of activity in the BIS and BAS systems.

For example, Schatz and Rostain (2006) found that comorbidity rates between anxiety and ADHD were as high as $25 \%$, in their review of the literature. If anxiety and ADHD are associated with high and low BIS functioning respectively, BIS functioning alone cannot account for the causes of these disorders.

Many of the more recent studies in this area assess BIS and BAS functioning through self-report measures that are correlated with brain functioning. The items often appear very similar to those from anxiety or impulsivity measures, therefore the association between anxiety and BIS functioning is not surprising. The overlap of these constructs suggests that research on BIS and BAS functioning can help researchers understand an individual's propensity to learn from reward and/or punishment without attempting to predict psychological disorders.

\section{Distinction between Anxiety and Sensitivity to Punishment}

Anxiety is defined by the DSM IV-TR (APA, 2000, pp. 820) as, "The apprehensive anticipation of future danger or misfortune accompanied by a feeling of dysphoria or somatic symptoms of tension. The focus of anticipated danger may be internal or external." This is a broad definition for a number of anxiety disorders outlined in the DSM IV-TR. Based on a factor analysis with a population of individuals with anxiety disorders, Zinbarg and Barlow (1996) proposed that each anxiety disorder has specific defining characteristics, but that there is a general factor across all anxiety disorders that they refer to as "negative affectivity." This negative affectivity could be what distinguishes someone who is more likely to avoid or escape from stimuli associated with negative consequences without excessive distress and impairment, and someone who qualifies for an anxiety disorder.

Although avoidance and escape serve an adaptive function, the persistence of these behaviors sometimes inhibits behavior that potentially yields positive outcomes or success. This interference is one distinction between healthy BIS functioning and an impairing anxiety disorder. McAllister, McAllister, Scoles and Hampton (1986) refer to avoidance as "fear reduction" because they speculated that conditioned stimuli that are paired with aversive consequences elicit fear and the avoidance behavior 
reduces that fear. In their work with hooded-rats, McAllister et. al. paired the activation of a light with shock and then taught subjects to escape the shock by jumping a hurdle into a safe compartment. The door to the safe compartment opened simultaneously with the onset of the shock. The rats were then given the opportunity to avoid the shock by opening the door with the onset of the light rather than the shock. All of the rats acquired the avoidance response and then the shocks were terminated until the avoidance response was extinguished in all subjects. The rats were then divided into three groups. For two groups, an extra pairing of the CS and the shock was administered. One of these groups was allowed to escape the shock and a second group was not. The third group did not receive an extra pairing. The next extinction was significantly slower for the group with extra pairing and the ability to escape. The negative reinforcement initially aided the rats to avoid aversive stimuli, but was maladaptive in the rats that had the extra pairing of the CS and the shock, once the aversive stimulus was removed. Individuals who have learned to avoid certain stimuli in their environment are more likely to continue to avoid situations that are associated with punishment or loss. In these rats there was no cost for avoidance but in some situations avoidance limits the opportunity for individuals to contact reinforcers. This research suggests a learning component for the development and maintenance of anxiety and avoidance.

\section{Anxiety and Performance}

Despite the propensity for anxious individuals to learn from negative reinforcement and punishment, anxiety is sometimes associated with poor performance. In a go/no-go discrimination task originally designed by Newman, Widom, and Nathan (1985), Yechiam et. al. (2006) measured omission, commission and consistency errors in a community sample of 400 adolescents. The attention to gains or loss, as defined by errors of omission and commission, were compared to elevated scores on externalizing, internalizing, or attention problems on the Child Behavior Checklist (CBCL; Achenbach, 1991a) or the Youth Self Report (YSR; Achenbach, 1991b), anxiety problems as indicated by Welsh Anxiety Scale (Welsh, 1956) or low intelligence based on the Wechsler Intelligence Scale for Children-Revised (WISCR; Wechsler, 1974). The task included 90 trials of two-digit numbers as stimuli that either yielded a win that was associated with winning money or a loss associated with money loss if the participant responded 
by pressing a button when presented with the number. Nothing happened if the participant did not press the button for both the winning and losing numbers. The participants were not given any indication of which numbers were associated with reward and which numbers were associated with loss. Participants had to learn which numbers were winning numbers based on trial and error.

Errors in responding to the stimuli were recorded as commission errors if the button was pressed in response to a losing number, omission errors if the button was not pressed in response to a winning number, and consistency errors were recorded when the same stimulus yielded different responses. Commission errors were significant for individuals who scored high on the YSR $(r=.11, \mathrm{p}<.05)$ and CBCL for externalizing disorders $(r=.18, p<.01)$, the CBCL for attention problems $(r=.16, p<.01)$, anxiety on the Welsh Anxiety Scale $(r=.13, p<.05)$ and low on IQ on the WISC-R $(r=-.24, p<.05)$. The only significant correlation with omission errors was for individuals who scored high on the CBCL for attention problems $(r=.13, p<.05)$. The most relevant findings for the present study were the consistency errors that were only significant for individuals who scored high on the Welsh Anxiety Scale $(r=-.10, p<.05)$ and individuals who scored low on the WISC-R $(r=.11, p<.05)$. The effect size for the correlation between anxiety and commission and inconsistency errors is very small and therefore less meaningful than the results for individuals with externalizing disorders. The link between anxiety and the propensity to learn from punishment was not supported by this study. It is unclear if the link was not supported because it does not exist, there were too many confounding variables, or if the individuals with an anxiety disorder made mistakes due to the distress associate with the task. Yechiam et. al. (2006) proposed that individuals high in anxiety "are more likely to find the task stressful and to detach themselves from it (and choose randomly) after a short experience."

Barlow (1991) hypothesized that anxiety is a future-oriented disorder that is uncontrollably focused on perceived threat. Barlow stated that the hypervigilance to future threats interferes with attention to task and thus negatively affects performance. The hypervigilance Barlow describes implies that individuals with anxiety disorders are more likely to attend or respond to stimuli associated with threats than individuals who have lower levels of anxiety. In that sense, hypervigilance is similar to 
Behavioral Inhibition 7

Gray's concept of sensitivity to punishment. In the go/no go task described previously, anxious

individuals could not benefit from hypervigilance because there were not stimuli associated with loss until after the initial presentation of a stimulus that resulted in loss. The sensitivity to this original punishment and the subsequent negative affectivity could be the source of the inconsistent responding.

\section{School Performance and Anxiety}

School is a setting where anxious individuals may learn avoidance and adaptive behaviors through sensitivity to punishment. Students who avoid talking in class are less likely to be subject to disciplinary action for being disruptive and students who complete their assignments are less likely to have their parents contacted about poor school performance. Despite the potentially beneficial effect of strong BIS functioning, anxiety even impairs school performance enough that it is one of three major subscales of the Child Anxiety Impact Scale-Parent Version (CAIS-P; Langley, Bergman, McCracken, \& Piacentini, 2004), that was designed to measure impairment associated with anxiety. The CAIS-P correlated significantly with Global Assessment of Functioning ratings $(\mathrm{r}=-.37, p<.05)$, ADIS-IV diagnostic severity scores $(\mathrm{r}=.31, p=.05)$ and CBCL internalizing scores $(\mathrm{r}=.45, p<.05)$ in a sample of children all diagnosed with an anxiety disorder (Langley et al., 2004). Children treated for anxiety disorders have increased school performance based on reduced CAIS-P scores after completing treatment (Wood, 2006). Thus, school performance is a potentially representative measure of the functional impairment associated with anxiety. However, since the effects of anxiety on school performance are difficult to examine in a laboratory, it is challenging to determine the effects of anxiety on school performance independent of the influence of other environmental or person variables. The relation between untreated anxiety and deficits in school functioning makes the task of differentiating between learning from punishment and impairing anxiety more important.

\section{Aversive Control}

Perone (2003) argues for the adaptive utility of aversive control stating, "It leads us to come in out of the rain, to blow on our hot coffee before we drink it, and to keep our fingers out of electrical sockets." Because individuals high in BIS functioning are more sensitive to negative reinforcement, it 
seems likely that negative reinforcement would be efficient in decreasing maladaptive avoidance. An example of maladaptive avoidance is a soccer player avoiding an open shot due to the fear of failure and negative evaluation. Coaches engage in negative reinforcement regularly to increase shooting by yelling, reducing playing time, and changing the structure of future practices. Decreasing avoidance of shooting will increase the likelihood of a goal and force the individual to be exposed to the fear-inducing situation. This form of aversive control increases the likelihood of shooting, opportunities for success and the extinction of the fear of failure and negative evaluation.

\section{Alternative Performance Tasks}

Because procedural learning has not been directly associated with the BIS, BAS or fight/flight systems, alternative performance tasks should be tested to determine if these systems are also associated with a sensitivity to punishment or reward on procedural learning tasks. Field and Davey (1997) designed an affective learning task that required subjects to distinguish between two novel prototypical faces based on a set of six characteristic features that were exactly opposite for the two prototypes. The two prototypes were labeled Martians and Venusians. The Martian prototype had antennae pointing straight up, a hair line slanting to the right, eyes close together, a long nose, a small ear, and a mouth near the bottom of its face. The Venusian prototype had the distinct features of antennae that pointed outward, hair sloping to the left, eyes far apart, a small pointy nose, a large ear and a high mouth. Subjects were presented with all 64 different combinations of features and told that images that had more Martian features than Venusians features were Martian and similarly, all images that had more Venusian than Martian features were Venusian. The varying levels of identifying characteristics lend themselves nicely to testing the difficulty of trials in a discrimination task. The novelty of the stimuli reduces the effects prior learning or associations that are intrinsic to using numbers as stimuli as compared to Yechiam et al.. (2006).

The present study used a computerized learning task that included the methods of a go/no-go task with more novel stimuli including the Martians and Venusians discussed above. The computerized learning task also incorporated an increase in aversive control between two phases to test its effects. 


\section{Hypotheses}

Behavioral Inhibition 9

\section{Research Question 1}

What type of errors will individuals with high behavioral inhibition and anxiety make in Phase One of the computerized learning task?

Hypothesis 1. Individuals with strong BIS functioning and higher levels of anxiety will make more omission errors than individuals with lower ratings of BIS functioning and anxiety.

Rationale. Gray (1970) found that individuals higher in BIS functioning learned better from punishment than individuals low in BIS functioning on a performance learning task. Since omission errors are not punished in Phase One, it is unlikely that individuals higher in BIS activity and anxiety will respond and increase their likelihood of punishment.

\section{Research Question 2}

What type of errors will individuals with high behavioral activation and impulsivity make in Phase One of the computerized learning task?

Hypothesis 2. Individuals with higher ratings of BAS functioning and impulsivity will have more commission errors than individuals who score lower on ratings of BAS functioning and impulsivity.

Rationale. Individuals with high BAS functioning and impulsivity have made commission errors as a result of reward sensitivity in previous studies. There is no reason to believe that the awareness of the contingencies of the computerized learning task will alter that behavior.

\section{Research Question 3}

How will increasing the aversive control affect the types of mistakes made on the task?

Hypothesis 3a. Individuals who rated higher on BIS functioning and anxiety will make fewer omission errors in Phase Two than in Phase One.

Rationale. The increased aversive control will offer more opportunities to learn from punishment and individuals higher in BIS functioning and anxiety will be more sensitive to that change.

Hypothesis $3 b$. BAS functioning and impulsivity will not be associated with a change errors between Phase One and Phase Two. 
Behavioral Inhibition 10

Rationale. There is not a change in the likelihood of reward between Phase One and Phase Two.

Individuals high in impulsivity and BAS functioning are not as likely to learn from punishment and therefore will have the same number of errors between Phase One and Phase Two.

Research question 4

How will the increase in aversive control affect the subjective ratings of negative affect?

Hypothesis 4. Increases in negative affect ratings between Phase One and Phase Two will increase with the level of BIS functioning and anxiety.

Rationale. Individuals higher in BIS functioning and anxiety are more sensitive to punishment and therefore will be more negatively affected by the change than individuals lower in BIS functioning and anxiety.

Method

\section{Participants}

Participants were 116 undergraduate students at West Virginia University between 18 and 25 years of age. Viable data were obtained for 109 participants (36 males and 73 females) due to seven individuals not acquiring the spacebar-pressing response in Phase One of the ACT.

Measures

Sensitivity to Punishment and Sensitivity to Reward Questionnaire (SPSRQ). The SPSRQ is a 48 item, yes-no scale (Turrubia, Avila, Molto \& Caseras, 2001) developed to measure the functioning of the BIS and BAS systems described by Gray's model of personality. The SPRSQ was chosen for the strong internal consistency of each subtest. The Sensitivity to Punishment (SP) subscale $(\alpha=.87)$ had a slightly stronger internal consistency than the Sensitivity to Reward (SR) subscale $(\alpha=.77)$. The test-retest correlation was .89 for SP and .87 for SR.

Beck Anxiety Inventory (BAI). The BAI (Beck, Epstein, Brown, \& Steer, 1988) is a 21-item selfreports that uses a four-point Likert-type scale designed to differentiate anxiety symptoms from depressive symptoms. It has strong internal consistency in a sample of individuals with outpatient anxiety 
disorders with $\alpha$ scores ranging from .85 to .93 (Steer, Ranieri, Beck, \& Clark, 1993). It has been

validated using a two-factor system of subjective and somatic anxiety (Hewitt \& Norton, 1993).

Positive and Negative Affect Scales (PANAS). The PANAS (Watson \& Clark, 1988) is a 20-item scale with two independent subscales to measure positive affect (PA) and negative affect (NA) on a fivepoint Likert -type scale. The internal consistency for the PA and NA subscales range from .88 to .90 and .84 to .87 respectively. The PANAS was chosen for its ability to measure affect over different time ranges including in the moment. The PANAS was administered by computer following each of the two phases described below to measure potential changes in affect between phases.

Barratt Impulsiveness Scale Version 11 (BIS-11). The BIS-11 (Stanford \& Barratt, 1995) a 30item self-report that utilizes a four-point scale ranging from 1 (rarely/never) to 4 (frequently/ always). It has three factors consisting of attention impulsiveness, motor impulsiveness and non-planning impulsiveness. The BIS-11 has strong internal consistency ranging between .79 and .83 for populations of undergraduates, substance-abuse patients, general psychiatric patients and prison inmates.

Aversive Control Task (ACT). The ACT is a computerized task designed for this thesis investigation that tests an individual's ability to identify novel stimuli independent of prior learning. The ACT is based on the go/no-go discrimination task used by Yechiam et al.. (2006). Modifications were made to address avoidance learning and test aversive control. The first change is that the ACT employs a modified set of the novel Martian and Venusian (MV) images from Field and Davey (1997) to eliminate the prior learning effects of using numbers. The modified images of the Martian and Venusian prototypes can be found in Appendix A and Appendix B respectively. As the ACT requires two phases, a second set of novel stimuli was counterbalanced between phases with the MV stimuli, to test for effects associated with the particular stimuli used. The second set of stimuli was comprised of Rocket and Shuttle prototypes with seven distinctive features and the 126 permutations of the rocket and shuttle features. The Rocket and Shuttle prototypes can be found in Appendix C and D respectively.

The first phase rewards correct identifications, penalizes commission errors, and has no effect for omission errors or correct rejections. In this phase participants learn to avoid incorrect responding and to 
Behavioral Inhibition 12

respond to rewarding stimuli. Phase Two changes the contingencies of responding (or not responding) slightly. Correct identifications, commission errors, and correct rejections are addressed the same as in Phase One, but omission errors are penalized the same as commission errors. Omission errors are associated with maladaptive avoidance and are the target of our intervention with aversive control.

At the beginning of Phase One, the computer displays the prototypes for the first set of stimuli and the following instructions: "The picture on the left is a Martian/Shuttle and the picture on the right is a Venusian/Rocket. Press Enter when you are done.” After the participant presses the Enter key, they are shown the following instructions and two sample pictures: "I am going to show you pictures that combine the features of Martians/Shuttles and Venusians/Rockets. If the picture has more features that are Martian/Shuttle than Venusian/Rockets like the image on the left, then it is a Martian/Shuttle. Press Enter to continue."

Once the participant presses Enter again they are shown the following instructions. "If you see a Martian/Shuttle, then press the spacebar. Every time you correctly identify a Martian, you will receive 5 points. Every time you press the space bar and there is a Venusian/Rocket picture, you will lose 5 points. Press Enter to begin."

After the participant presses the Enter key again, they are shown the first of the 126 potential combinations of the stimuli excluding the prototypes. The image will be displayed for 1.5 seconds during which the participant can respond by pressing the spacebar. If the participant correctly identifies a Martian/Shuttle then they are shown a screen that displays " +5 ". If the participant correctly rejects a Venusian/Rocket or misses a Martian/Shuttle (does not press the spacebar) then, they are shown a screen that displays " +0 ". If the participant incorrectly responds to a Venusian/Rocket by pressing the spacebar, then the screen displays "-5". The number of features representative of each stimulus's membership class, or the difficulty of each trial, is taken into account. Because there are seven features, then it takes at least four features to have a dominant set of features. Therefore, the difficulty is broken into four features, five features, and six features of the items membership class. There are 14 items that have six features, 42 items that have five features, and 70 items that have four features of the membership class. The items are 
Behavioral Inhibition 13 shown randomly in blocks of nine that are in proportion to their frequency in the population. This includes one six-feature item, three five-feature items, and five six-feature items. All of the combinations of features will be shown in individual trials in Phase One. After all of the stimuli have been presented, the participant completes a computer-administered Positive Affect and Negative Affect Scale (PANAS) self-report measure.

After completing the PANAS, the same procedure is repeated in Phase Two with two changes. The first change is that a different set of stimuli is used, but they have the same number of features and trials. The second change, is that omissions are penalized by displaying "-5" with each miss of a target stimulus. After all of the stimuli in the second set are presented, the participant completes the computerized PANAS ratings again.

Types of errors (omission and commission) are the variables of primary interest. In addition to error scores, each participant receives an overall performance score for each phase that represents success on the task. In Phase One, the Total score is calculated by adding one point for a correct identification and subtracting a point for each commission error. In Phase Two, the Total score is calculated by adding one point for a correct identification, subtracting a point for each commission error, and subtracting a point for each omission error. The Final Total score is calculated by summing the Phase One Total score and the Phase Two Total Score. Total scores are representative of the feedback that the participants receive during the task.

To determine the effects of the increase in aversive control through negative reinforcement, change scores are calculated for omission and commission errors by subtracting the number of errors in Phase One from the number of errors in Phase Two. Total scores for each phase cannot be compared because there are twice as many opportunities to lose points in Phase Two than in Phase One.

\section{Procedure}

Participants were recruited from undergraduate psychology courses and scheduled for individual sessions in the lab using SONA System's web-based human subject management software. Upon arriving in the lab, participants were given an informed consent to read. After participants signed the informed 
Behavioral Inhibition 14 consent, the primary investigator or a research assistant administer the following questionnaires: Sensitivity to Punishment and Sensitivity to Reward Questionnaire, Beck Anxiety Inventory, Social Phobia and Anxiety Inventory, Barratt Impulsiveness Scale Version 11, and Beck Depression Inventory II. After completing the self-report questionnaires, participants were told about the Aversive Control Task (ACT) and that each point the participant earned equaled an entry into a lottery for $\$ 100$. The investigator then initiated the ACT program and left the room once the screen displaying the instructions was visible, closing the door behind them. Participants were instructed to open the door once the task was completed.

\section{Results}

Descriptive statistics are presented in Table 1. Preliminary analyses determined that there were gender differences on some of the performance variables. One-way ANOVAs determined that females made more commission errors $(M=23.9, S D=9.9)$ than males $(M=18.7 S D=10.3)$ in Phase One $F$ $(1,107)=6.61, p<.02$. Similarly, males earned higher scores in Phase One $F(1,107)=4.95, p<.03$ with a mean score of $13.1(S D=6.4)$ than females who earned a mean score of $9.5(S D=8.6)$. Males also had a larger increase in commission errors between phases one and two $F(1,107)=7.97, p<.01$ with a mean increase of $12.2(S D=11.1)$ compared to a mean increase of $5.7(S D=11.3)$ for women. No other gender differences were found for self-report or performance measures.

\section{Task Validation}

A repeated measures ANOVA shows a significant decrease in omission errors between Phase One $(M=30.1, S D=9.8)$ and Phase Two $(M=22.0 . S D=7.6), F(1,107)=82.55, p<.001$. There also was a significant increase in commission errors between Phase One $(M=22.2, S D=10.3)$ and Phase Two $(M=30.2, S D=10.8), F(1,107)=50.76, p<.001$. No significant differences in the change in commission or omission errors were found based on the order of administration of ACT stimuli. The increase in responding in Phase Two shows that the contingency change between Phase One and Phase Two negatively reinforced responding regardless of whether the response was a correct identification or a commission error. 
Alien and Ship Stimuli Order Effects

Behavioral Inhibition 15

Performance results by stimuli are presented in Table 2. One-way ANOVAs indicated that there were some differences associated with the order of administration of the Ship and Alien stimuli. Participants who were shown the Alien stimuli in Phase Two $(M=32.4, S D=11.2)$ committed more commission errors than participants who were shown the Ship stimuli in Phase Two $(M=28.2, S D=$ 10.2), $F(1,107)=4.20, p<.05$. Individuals shown the Alien stimuli in Phase Two earned a mean score of -13.8 $(S D=10.9)$ in Phase Two which was significantly lower than individuals shown the Ships in Phase Two $(M=-8.25, S D=11.3), F(1,107)=6.79, p=.01$. Similarly, Individuals shown the Alien stimuli in Phase Two had lower overall scores $(M=-3.63, S D=14.2)$ than individuals shown the Ships in Phase Two $(M=2.8, S D=14.3), F(1,107)=5.52, p<.03$. No other differences were observed between the stimuli or the order of administration.

\section{Correlations among study variables}

Table 3 shows a complete correlation matrix for all study. Unsurprisingly, there was a significant positive correlation between Sensitivity to Punishment and BAI scores $(r=.30, p=001)$. Similarly, there was a significant positive correlation between Sensitivity to Reward and BIS-11 scores $(r=.36, p<.001)$. One surprising finding was that BAI and BIS-11 scores were significantly positively correlated $(r=.35, p$ $<.001)$ indicating that individuals who rated themselves higher in anxiety also rated themselves higher in impulsivity.

There were significant correlations among performance measures on the ACT. Phase One omission errors were significantly correlated with Phase Two omission errors $(r=.46, p<.001)$ indicating that individuals who made more omission errors in Phase One were also made more omission errors in Phase Two. Phase One commission errors were significantly positively correlated with Phase Two commission errors $(r=.37, p<001)$ further indicating that individuals made the same types of errors in both phases.

Phase One omission errors were negatively correlated with Phase One commission errors $(r=$ $.67, p<.001)$ and Phase Two commission errors $(r=-.35, p<.001)$. Phase Two omission errors also 
Behavioral Inhibition 16

were negatively correlated with Phase One commission errors $(r=-.41, p<.001)$ and Phase Two

commission errors $(r=-.67, p<.001)$. These findings suggest that individuals were consistently more

likely to make one kind of error than the other. The only significant correlation with an increase in

negative affect between Phase One and Phase Two was an increase in commission errors between the two phases $(r=.26, p<.01)$.

Predicting task performance by self-report measures

Three standard linear regressions were done to predict omission errors, commission errors and overall performance scores in Phase One based on SP scores. Three standard linear regressions were performed to predict omission errors, commission errors and overall performance scores from Phase One based on BAI scores. BAI and SP scores were not predictive of omission errors, commission errors, or overall performance scores for Phase One (see Table 4).

Two standard linear regressions were performed to predict the change in omission errors between phases based on SP scores and BAI scores. Neither SP nor BAI scores were predictive of a change in omission errors between Phase One and Phase Two (see Table 4).

Two standard linear regressions were completed to predict the change in negative affect between phases based on SP scores and BAI scores. Neither SP nor BAI scores were predictive of a change in negative affect ratings between Phase One and Phase Two (see Table 4).

Three standard linear regressions were conducted to predict omission errors, commission errors, and overall performance scores from Phase One based on SR scores. Three standard linear regressions were also conducted to predict omission errors, commission errors, and overall performance scores from Phase One based on scores on the BIS - 11. SR and BIS-11 scores were not predicted of omission errors, commission errors, or overall performance scores in Phase One (see Table 5).

Two standard linear regressions were completed to predict the change in omission errors based on SR and BIS-11 scores. Two standard linear regressions were completed to predict the change in commission errors between phases based on SR and BIS-11 scores. SR and BIS-11 scores were not predictive of a change in omission or commission errors (see Table 5) 


\section{Discussion}

The primary goal of this study was to examine the effects of aversive control on individuals with higher anxiety or sensitivity to punishment. While this objective has not been accomplished, the ACT task was determined to be able to manipulate responding such that the direction of the effect was as expected for both omission and commission errors. There are several factors related to the ACT and the study methods that potentially compromised the ability to determine individual differences. Previous findings indicate that anxiety is not predictive of omission or commission errors, but rather is related to inconsistent responding to stimuli (Yechiam et. al, 2006). The present study replicated the lack of association between types of errors on go/no-go tasks and anxiety, but the single presentation of stimuli in the ACT did not allow for a measure of inconsistent responding. Unfortunately, the study did not replicate the findings that individuals higher in impulsivity were more likely to commit commission errors. The inability of the first phase of the ACT to replicate previous findings on go/no-go tasks suggests that there may be problems with the task construction that make it insensitive to individual differences in impulsivity.

There also may have been a difference between self-report measures and the corresponding response styles of participants. The data suggests that individuals were more likely to make the same type of errors in Phase Two that they did in Phase One. The data also shows a negative relation between omission and commission errors suggesting that individuals were more likely to make one kind of error instead of the other. These data suggest that participants had set response styles throughout the task. The types of self-report measures used may not have been sensitive to the subtle differences between individuals such that they would reflect their response styles on the ACT. The BAI, for example, consists primarily of physiological symptoms that may not be the most indicative measure of an inhibited response style but indicative of impairing levels of anxiety.

It is impossible to determine which variables in the ACT, produced the differences between the results for this study and other go/no-go tasks due to the range of differences. The potential nuisance 
variables include the difficulty of the task, the acquisition of responses, and the abstract nature of the rewards and losses on the task. The ACT was potentially difficult for participants due to the complexity of the stimuli and the short duration of stimulus presentation. The Alien faces and Ships had seven distinct features and were potentially difficult to distinguish even with an unlimited amount of time. Only by counting four features from one stimulus group could a participant know for sure whether a response would be rewarded or punished. The fast pace of the task was chosen because of the ability to count the number of similar features and determine precisely which group each stimulus belonged to. Unlimited time would make the task a better measure of memory rather than a measure of inhibition and impulsivity. Participants may have made associations with features that were not consistent with the overall consequence of responding to each stimulus. The potential pairing between the nonreward features and reward instead of a set of features from the reward group, was highly likely due to the presence of at least one nonreward feature in each occurrence of reward. Phenomena like learned helplessness could occur when individuals receive punishment during the presentation of features they had come to associate with reward. The same learning may have occurred with features of rewarding stimuli that occurred with stimuli that had more nonreward features.

The presence of individuals in the sample, who were unable to produce the target response of spacebar-pressing, indicates that there may have been others who acquired the response during the task rather than in the rule-governed fashion intended by the researcher. All of the individuals who did not press the space bar in Phase One, did press the space bar in Phase Two after the directions had been presented a second time suggesting that they did not read the instructions before initiating Phase One. Theoretically, impulsive individuals would be more likely to begin the task without reading the instructions. The unusual lack of a correlation between commission errors and impulsivity may have been due to impulsive individuals having a delayed acquisition of the target response and consequentially having a depressed number of commission errors.

Another concern about the task was the strength of the rewards and losses in the ACT. Participants were alerted when they made gains or losses, but the actual likelihood of obtaining a real 
Behavioral Inhibition 19

rather than an abstract reward was less than $1 \%$. The lack of a true reward had even less of an impact on the target of the study than the lack of true losses or punishment. Avoidance of punishment or loss was nonexistent in this study because the only negative consequence was a reduced probability of reward. The study did not provide any reasonable consequence to motivate individuals higher in anxiety or sensitivity to punishment to respond to stimuli in the ACT. The researchers observed the participants complaining about not performing as well during Phase Two as they had in Phase One, which indicates that the change in consequences may have been aversive but not in a meaningful way for the participants.

Finally, the presence of order effects for the presentation of the ACT sets of stimuli indicates that the two sets of stimuli were not similar enough in difficulty to be used interchangeably. It is interesting that the differences only occurred in Phase Two, which narrows the possible explanations. The first explanation is that the Alien faces were harder to discriminate than the Ships. The second possible explanation is that the differences only occurred in Phase Two because the target response had not been developed in Phase One sufficiently to detect order effects.

The lack of change in negative affect or performance errors despite the increase in aversive control is not surprising due to the extensive complications associated with the ACT. One explanation is that a stable baseline was not obtained before changing the contingencies due to superstitious learning or a delayed acquisition of the target response. Another explanation is the lack of motivation to perform well mediated by the lack of opportunity for loss and the remoteness of the opportunity for a true reward. No matter which aspect or interaction of aspects undermined the construct validity of the ACT, in its current form it has not proven to be a useful measure of the effects of aversive control. Several changes should be made if the ACT is to be used for research purposes in the future. The length of stimulus presentation should be studied parametrically to determine whether the speed of the task mediated the construct validity of the task. The acquisition of the target response should be trained with an unrelated task prior to initiating the task to prevent the effects of acquisition and shaping from impacting the outcome variables. The stimuli should be modified to eliminate order effects between the Alien and Ship sets of stimuli. It is possible that simpler discriminative stimuli could be used, such as the features of 
Behavioral Inhibition 20 natural concepts used by Hernstein, Loveland, and Cable (1976). These researchers trained pigeons to keypeck in response to photos with certain features like trees and to not peck when presented with photos that did not contain trees. This study aimed to use stimuli that would not be familiar to participants, but the discrimination of stimuli in the ACT seemed to be too difficult for participants. Using previously established go/no-go tasks may be useful to study aversive control if testing effects could be minimized. Aversive control requires establishing a baseline before implementing the aversive control and examining the effects of the contingency change. The present task attempted to establish a baseline but still failed to have a reversal of the experimental manipulation. The inconsistency between the groups of stimuli suggests that implementing a meaningful reversal would be even more difficult.

Aside from the complications of the ACT, aversive control is very difficult to study in human populations due to the need for a real cost to participants. Future studies could use aversive techniques such as shock to study aversive control but such studies would have difficulty recruiting and consenting the target population of this study, individuals high in anxiety. Avoidance does not begin at the door to the research laboratory, but is an integral part of these individuals' day to day lives. A second possibility is providing research participants with baseline level of compensation for participation, and having losses on the task result in a decrease in the baseline level of compensation. This second suggestion is still a contrived means of implementing loss, but is slightly more ecologically valid than the lottery entries utilized in the present study.

Despite the difficulties, it is important that researchers continue to study the effects of aversive control in human populations. The technique is used in many legal and educational settings as a means to induce compliance to correct behaviors ranging from truancy to paying taxes. It is unclear how aversive negative reinforcement differentially effects individuals in society and will continue to be elusive until researchers develop an ecologically valid paradigm to study its effects. Most of the domains where negative reinforcement is utilized implement its use legislatively and therefore universally. The universal application of negative reinforcement obscures the true effects by not establishing a clear baseline and not having any kind of control group for data that can only be analyzed by comparing rates or averages. 
Behavioral Inhibition 21

Hopefully this failed attempt at measuring the effects of aversive control will inspire studies with improved experimental control to determine its effects. 
Achenbach, T. M. (1991a). Manual for the Child Behavior Checklist and 1991 profile. Burlington, VT: University of Vermont, Department of Psychiatry.

Achenbach, T. M. (1991b). Manual for the Youth Self-Report and 1991profile. Burlington, VT: University of Vermont, Department of Psychiatry.

American Psychiatric Association. (APA). (2000). Diagnostic and Statistical Manual of Mental Disorders ( $4^{\text {th }}$ ed., text revision). Washington, DC: Author.

Barlow, D.H. (1991). Disorders of emotions: clarifications, elaborations and future directions. Psychological Inquiry, 2, 97-105.

Beck, A.T., Epstein, N., Brown, G., \& Steer, R.A. (1988). An inventory for measuring clinical anxiety: Psychometric properties. Journal of Consulting and Clinical Psychology, 56, 893-897.

Carver, C.S., \& White, T.L. (1994). Behavioral inhibition, behavioral activation, and affective responses to impending reward and punishment: The BIS/BAS Scales. Journal of Personality and Social Psychology, 67, 319-333.

Corr, P.J., Pickering, A.D., \& Gray, J.A. (1997). Personality, punishment, and procedural learning: A test of J.A. Gray's anxiety theory. Journal of Personality and Social Psychology, 73, 337-344.

Field, A.P., \& Davey, G.C.L., (1997). Conceptual conditioning: Evidence for an artifactual account of evaluative learning. Learning and Motivation, 28, 446-464.

Fowles, D.C., (1980). The three arousal model: Implication of Gray's two-factor learning theory for heart rate, electrodermal activity, and psychopathy. Psychophysiology, 17, 87-104.

Gray, J.A. (1970). The psychophysical basis of introversion-extraversion. Behaviour Research and Therapy, 8, 249-266.

Gray, J.A. (1978). The neuropsychology of anxiety. British Journal of Psychology, 69, 417-434.

Gray, J.A. (1982). The Neuropsychology of Anxiety: An Enquiry into the Functions of Septo-Hippocampal System. Oxford: Oxford University Press.

Gray, J.A. (1987). Neuropsychology of emotion and personality. Cognitive Neurochemistry, New York, 
NY: Oxford University Press.

Harmon-Jones, E., \& Allen, J.J.B. (1997). Behavioral activation sensitivity and resting frontal EEG asymmetry: Covariation of putative indicators related to risk for mood disorders. Journal of Abnormal Psychology, 106, 159-163.

Hernstein, R. J., Loveland, D. H., \& Cable, C. (1976). Natural concepts in pigeons. Journal of Experimental Psychology: Animal Behavior Processes, 2, 285-302.

Hewitt, P.L., Norton, G.R. (1993). The Beck Anxiety Inventory: A psychometric analysis. Psychological Assessment, 5, 408-412.

Langley, A.K., Bergman, R.L., McCracken, J., \& Piacentini, J.C. (2004). Impairment in childhood anxiety disorders: Preliminary examination of the child anxiety impact scale -parent version. Journal of Child and Adolescent Psychopharmacology, 14, 105-114.

McAllister, W.R., McAllister, D.E., Scoles, M.T., \& Hampton, S.R. (1986). Persistence of fear-reducing behavior: Relevance for the conditioning theory of neurosis. Journal of Abnormal Psychology, 95, 365-372.

Newman, J.P., MacCoon, D.G., Vaughn, L.J., \& Sadeh, N. (2005). Validating a distinction between primary and secondary psychopathy with measures of Gray's BIS and BAS constructs. Journal of Abnormal Psychology, 114, 319-323.

Newman, J. P., Widom, C. S., \& Nathan, S. (1985). Passive avoidance in syndromes of disinhibition, psychopathy, and extraversion. Journal of Personality and Social Psychology, 48, 1316-1327.

Perone, M. (2003). Negative effects of positive reinforcement. The Behavior Analyst, 26, 1-14.

Quay, H.C. (1997). Inhibition and attention deficit hyperactivity disorder. Journal of Abnormal Child Psychology, 25, 7-14.

Schatz, D.B., \& Rostain, A.L. (2006). ADHD with comorbid anxiety. Journal of Attention Disorders, 10, 141-149.

Shapiro, S.K., Quay, H.C., Hogan, A.E., \& Schwartz, K.P. (1988). Response perseveration and delayed 
Behavioral Inhibition 24

responding in undersocialized aggressive conduct disorder. Journal of Abnormal Psychology, 97, 371-373.

Stanford, M.S., \& Barratt, E.S. (1995). Factor structure of the Barratt Impulsiveness Scale. Journal of Clinical Psychology, 51, 768-775.

Steer, R.A., Ranieri, W.F., Beck, A.T., \& Clark, D.A. (1993). Further evidence for the validity of the Beck Anxiety Inventory with psychiatric outpatients. Journal of Anxiety Disorders, 7, 195-205.

Torrubia, R., Avila, C., Molto, J., \& Caseras, X. (2001). The Sensitivity to Punishment and Sensitivity to Reward Questionnaire (SPSRQ) as a measure of Gray's anxiety and impulsivity dimensions. Personality and Individual Differences, 31, 837-862.

Watson, D. \& Clark, L. A. (1988). Development and validation of brief measures of positive and negative affect: The PANAS scales. Journal of Personality and Social Psychology, 54, 1063-1070.

Wechsler, D. (1974). Wechsler Intelligence Scale for Children-Revised. New York, NY: Psychological Corporation.

Welsh, G. (1956). Factor dimensions A and R. In G. S. Welsh \& W. G. Dahlstrom (Eds.), Basic readings on the MMPI in psychology and medicine (pp. 264-281). Minneapolis, MN: University of Minnesota Press.

Wood, J. (2006). Effect of anxiety reduction on children's school performance and social adjustment. Development Psychology, 42, 345-349.

Yechiam, E., Goodnight, J., Bates, J.E., Busemeyer, J.R., Dodge, K.A., Petit, G.S., \& Newman, J.P. (2006). A formal cognitive model of the go/no-go discrimination task: Evaluations and implications. Psychological Assessment, 18, 239-249.

Zinbarg, R.E., \& Barlow, D.H. (1996). Structure of anxiety and the anxiety disorders: A hierarchical model. Journal of Abnormal Psychology, 105, 181-193. 
Behavioral Inhibition 25

Table 1

Descriptive Statistics for Assessment and Performance Measures

\begin{tabular}{lrrrr}
\hline & $M$ & $S D$ & Min & Max \\
\hline Sensitivity to Punishment & 10.77 & 5.24 & 1 & 23 \\
BAl & 10.61 & 4.03 & 0 & 36 \\
Sensitivity to Reward & 12.48 & 8.33 & 2 & 25 \\
BIS-11 & 67.57 & 8.91 & 44 & 92 \\
Phase One Omission Errors & 30.11 & 9.82 & 11 & 56 \\
Phase One Commission Errors & 7.84 & 11.58 & 2 & 49 \\
Phase One Total Score & 10.69 & 8.13 & -14 & 26 \\
Phase Two Omission Errors & 22.02 & 7.58 & 3 & 46 \\
Phase Two Commission Errors & 30.24 & 10.83 & 4 & 63 \\
Phase Two Total Score & -10.99 & 11.36 & -41 & 16 \\
Omission Change & -8.10 & 9.25 & -37 & 11 \\
Commission Change & 7.84 & 11.58 & -20 & 36 \\
Negative Affect Change & 1.19 & 3.31 & -7 & 12 \\
\hline
\end{tabular}


Behavioral Inhibition 26

Table 2

Descriptive Statistics by ACT Stimuli

\begin{tabular}{lcccccc}
\hline & \multicolumn{2}{c}{ Omission Errors } & \multicolumn{2}{c}{ Commission Errors } & \multicolumn{2}{c}{ Total Scores } \\
& $M$ & $S D$ & $M$ & $S D$ & $M$ & SD \\
\hline Phase One Aliens & 30.36 & 8.90 & 23.00 & 11.53 & 11.22 & 7.47 \\
Phase One Ships & 29.85 & 10.76 & 23.00 & 11.53 & 10.15 & 8.79 \\
Phase Two Aliens & 22.13 & 7.49 & 32.35 & 10.15 & -10.99 & 11.36 \\
Phase Two Ships & 21.91 & 7.74 & 28.16 & 11.16 & -8.25 & 11.27 \\
\hline
\end{tabular}


Table 3

Correlations among Primary Study Variables

\begin{tabular}{|c|c|c|c|c|c|c|c|c|c|c|c|}
\hline & 1 & 2 & 3 & 4 & 5 & 6 & 7 & 8 & 9 & 10 & 11 \\
\hline 1. Sensitivity to Punishment & & .01 & $.30 * *$ & -.01 & -.02 & -.09 & .08 & .01 & .05 & .01 & .14 \\
\hline 2. Sensitivity to Reward & & & .13 & -.01 & -.02 & .00 & .01 & .01 & .03 & .02 & $.36 * *$ \\
\hline 3. BAI & & & & -.09 & .11 & -.12 & .18 & .00 & .08 & .08 & $.35^{* *}$ \\
\hline 4. Phase 1 Misses & & & & & $.67 * * *$ & $.46 * * *$ & $-.35 * * *$ & $-.68 * * *$ & $.24 *$ & -.06 & -.05 \\
\hline 5. Phase 1 False Alarms & & & & & & $-.41 * * *$ & $.37 * * *$ & $.38 * * *$ & $-.52 * * *$ & -.11 & -.06 \\
\hline 6. Phase 2 Misses & & & & & & & $-.67 * * *$ & $.34 * * *$ & $-.26 * *$ & -.03 & -.04 \\
\hline 7. Phase 2 False Alarms & & & & & & & & -.18 & $.57 * * *$ & .14 & -.08 \\
\hline 8. Change in Misses & & & & & & & & & $-.47 * * *$ & .05 & .02 \\
\hline 9. Change in False Alarm & & & & & & & & & & $.26 * *$ & -.03 \\
\hline 10. Change in Negative Affect & & & & & & & & & & & .07 \\
\hline 11. BIS - 11 & & & & & & & & & & & \\
\hline
\end{tabular}

$* p<.05 . * * p<.01 . * * * p<.001$. 
Behavioral Inhibition 28

Table 4

Linear Regressions for performance measures by Sensitivity to Punishment and BAI Scores

\begin{tabular}{lcccc}
\hline & \multicolumn{3}{c}{ SP } & \multicolumn{3}{c}{ BAI } \\
& $\beta$ & $R^{2}$ & $\beta$ & $R^{2}$ \\
\hline P1 Omission & -.076 & .01 & -.089 & .01 \\
P1 Commission & -.024 & .00 & .109 & .01 \\
P1 Total Score & .122 & .02 & -.029 & .00 \\
Omission Change & .007 & .00 & -.004 & .00 \\
NA Change & .012 & .00 & .079 & .01 \\
\hline
\end{tabular}


Table 5

Linear regressions for performance measures by Sensitivity to Reward and BIS-11 scores

\begin{tabular}{lrccc}
\hline & & SR & \multicolumn{2}{c}{ BIS-11 } \\
& $\beta$ & $R^{2}$ & $\beta$ & $R^{2}$ \\
\hline P1 Omission & -.007 & .00 & -.049 & .00 \\
P1 Commission & -.018 & .00 & -.055 & .00 \\
P1 Total Score & .031 & .00 & .128 & .02 \\
Omission Change & .009 & .00 & .020 & .00 \\
Commission Change & .032 & .00 & -.026 & .00 \\
\hline
\end{tabular}


Behavioral Inhibition 30

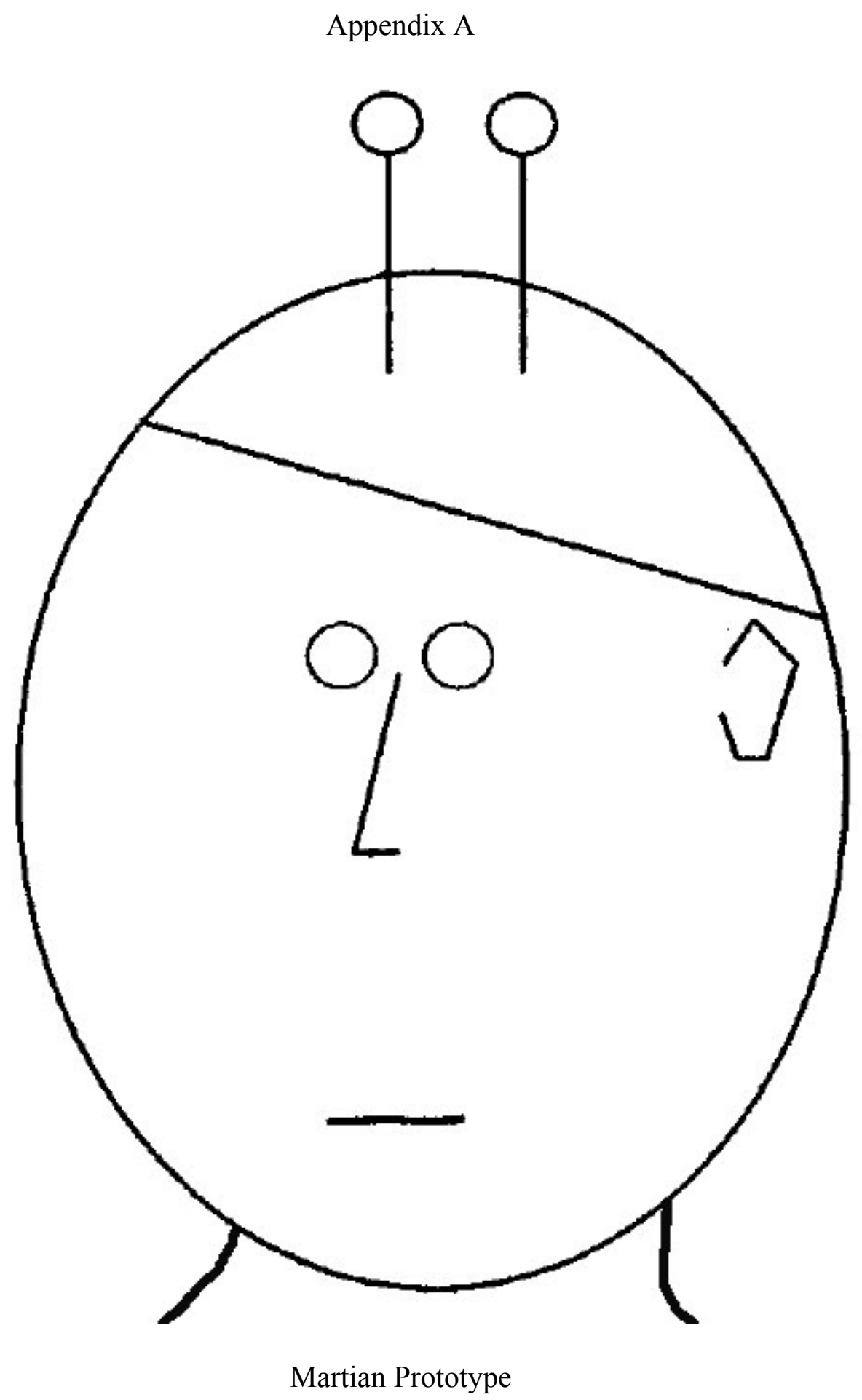


Behavioral Inhibition 31

Appendix B

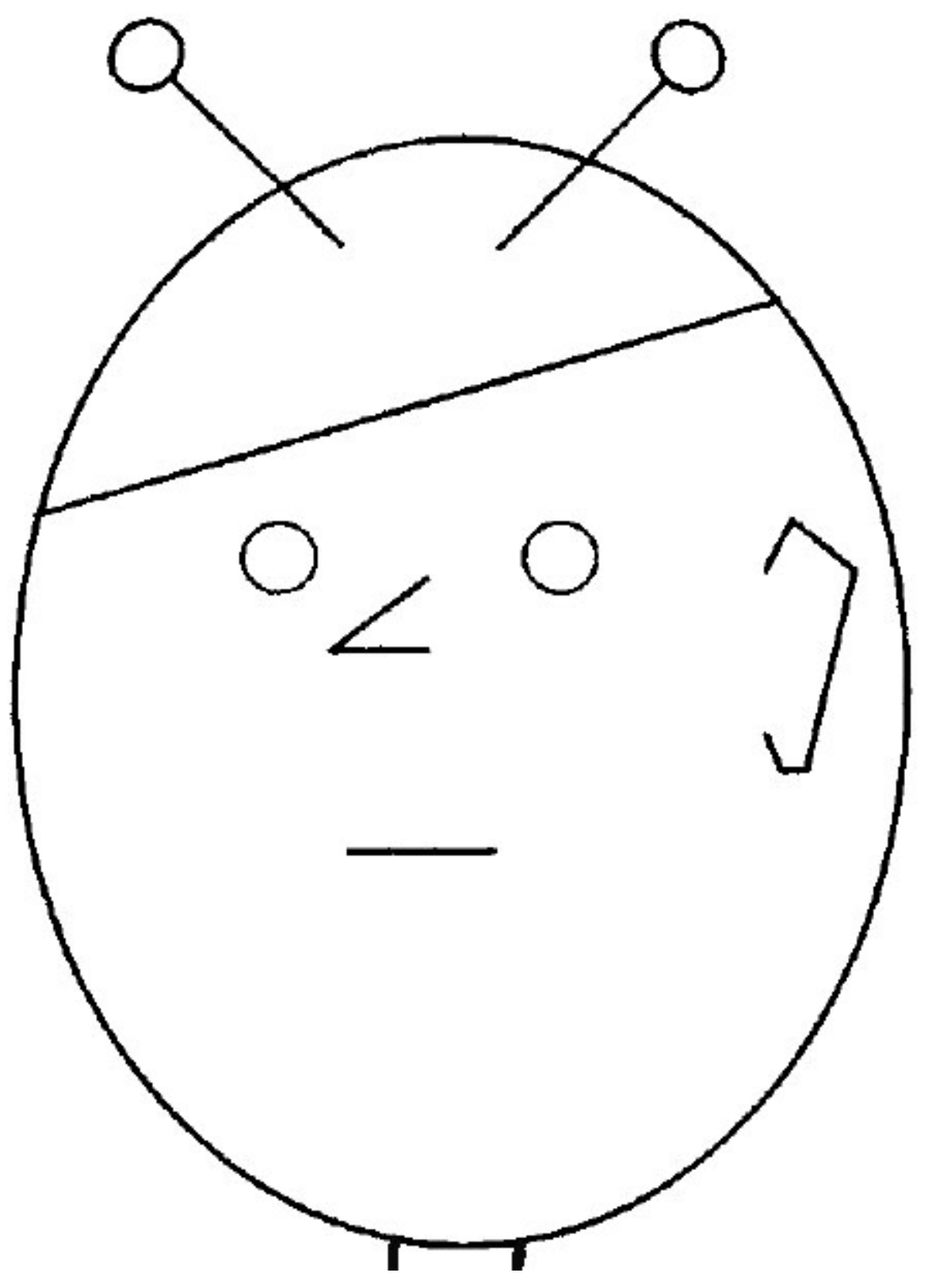

Venusian Prototype 
Behavioral Inhibition 32

Appendix C

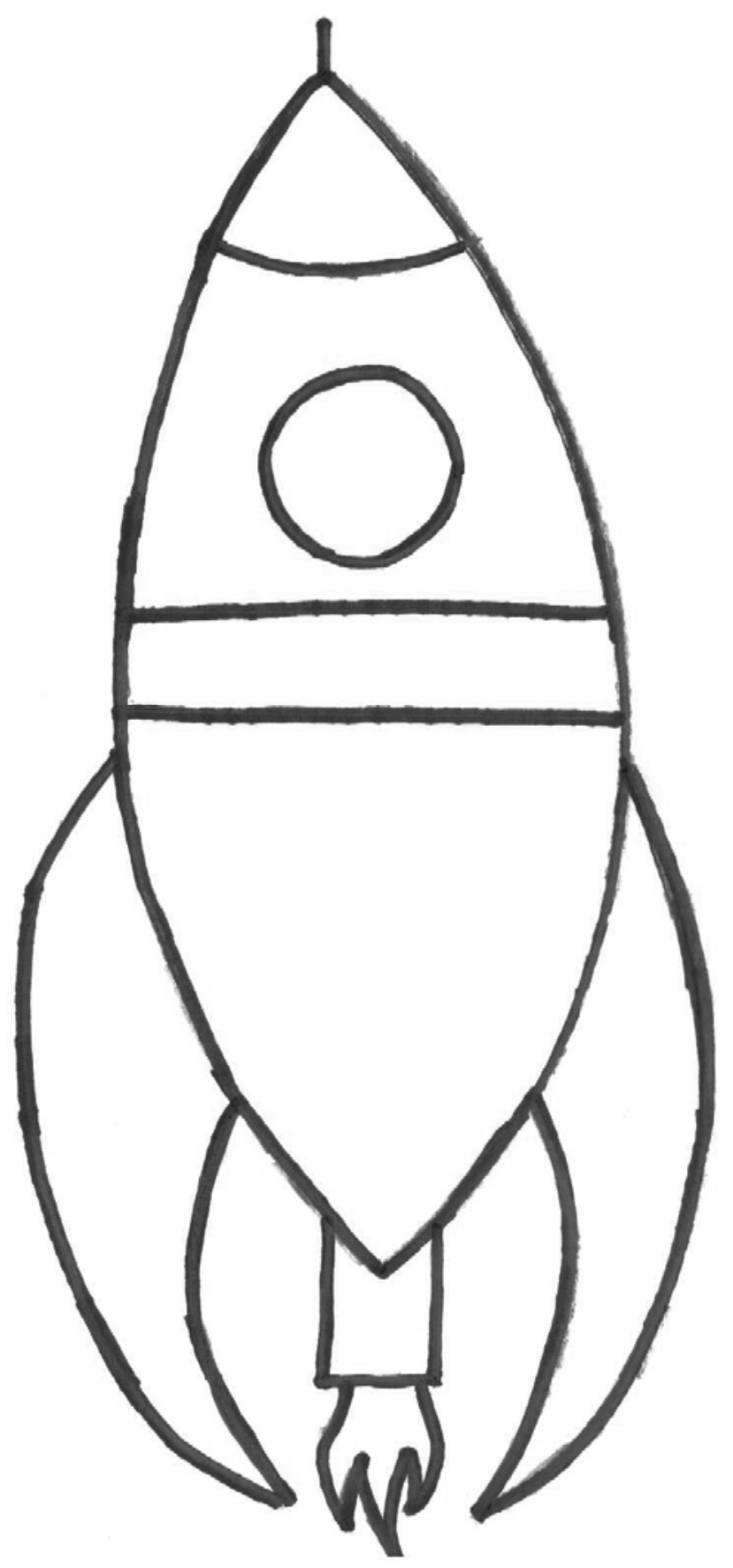

Rocket Prototype 
Behavioral Inhibition 33

Appendix D

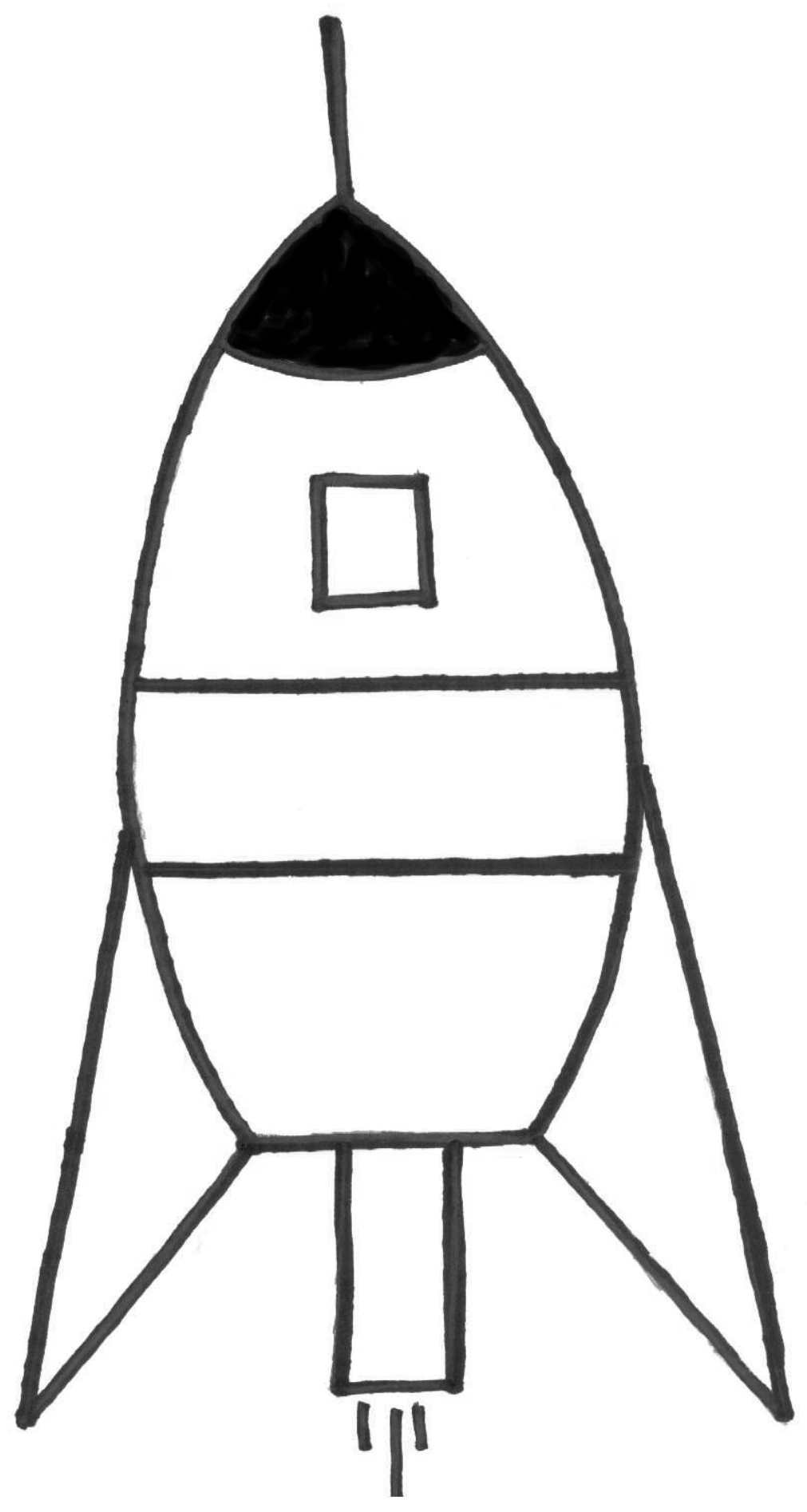

Shuttle Prototype 


\title{
Curriculum Vitae
}

\author{
Chad Brice \\ 535 Woodland Circle, Morgantown, WV 26505 \\ (919)923-2802 chad.brice@mail.wvu.edu
}

\section{Education:}

International Baccalaureate Degree, North Mecklenburg High School, Huntersville, North Carolina, August 1995-June 1999.

B.A. Psychology, University of North Carolina at Chapel Hill, Chapel Hill, North Carolina, August 1999-July 2003.

\section{Teaching Experience:}

Introduction to Psychology, Fall 2006 and Spring 2007, West Virginia University, Supervisor: Cheryl McNeil PhD

Clinical Interviewing, Fall 2008, West Virginia University, Teaching Assistant, Supervisor: Elisa Krackow PhD

Undergraduate Research Supervision, Chris Drescher, Undergraduate Research Assistant, Spring 2008 - Spring 2009

Supervised data collection and writing an undergraduate honors thesis.

\section{Publications:}

Ale, C.M., Chorney, D.C., Brice, C.S., \& Morris, T.M. (submitted). Facial Affect Recognition and Social Anxiety in Preschool Children. Submitted to Early Child Development and Care on January 13, 2009.

\section{Presentations:}

Moore, P., Garcia, A., Gonzalez, A., Brice, C., Thienemann, M., Huffman, L. Physiological Arousal in Parent-Child Interaction Paradigms Commonly Used in Anxiety Research. Poster Presentation. Society for Research in Child Development. April 2005. Atlanta, GA.

Brice, C., Jones, A., Ale, C., Drescher, C., \& Morris, T. Actions Speak Louder than Words: An Analysis of ABCT Poster Methodology. Poster Presentation. Association for Behavioral and Cognitive Therapies. November 2008. Orlando, FL.

Ale, C., Chorney, D., Brice, C., \& Morris, T. Social Anxiety and Facial Affect Recognition in Preschool Children. Poster Presentation. Association for Behavioral and Cognitive Therapies. November 2008. Orlando, FL. 


\section{Workshops:}

Interpersonal Therapy for Depression, Greg Hinrichsen PhD, April 14, 2007, WVU. Employment:

July 2008-Present Psychological Intern

Washington County Children and Youth Services, Washington, Pennsylvania

Supervisor: Michael Crabtree PhD

May 2007-July 2008 Graduate Assistant, Quinn Curtis Center

Department of Psychology, West Virginia University, Morgantown, West Virginia

Supervisor: William Fremouw PhD

Responsibilities included screening potential clients, conducting intakes and evaluations, and managing quality assurance checks.

Aug 2006-May2007 Graduate Teaching Assistant, Introduction to Psychology

Department of Psychology, West Virginia University, Morgantown, West Virginia

Supervisors: Cheryl McNeil PhD and Claire St. Peter-Pipkin

Mar 2006-July 2006 Research Assistant, Cognitive Behavioral Therapy for Relapse Prevention (CBT-RP)

Department of Psychiatry, Duke University Medical Center, Durham, North Carolina

Supervisor: David Goldston PhD

Responsible for administering Peabody Picture Vocabulary Tests, urine drug screens, conducting self-reports with patients and assisting in grant and IRB submissions.

June 2004-July 2006 Research Assistant, Pediatric OCD Treatment Study (POTSII) Department of Psychiatry, Duke University Medical Center, Durham, North Carolina

Supervisors: John March MD, MPH

Phoebe Moore PhD

Responsible for recruitment, conducting self reports with patients, cleaning data, data entry, facilitating cross-site collaboration, scheduling visits, preserving the blind and creating case report forms.

June 2004-July 2006 Research Assistant, OCD Imaging Pilot 
Department of Psychiatry, Duke University Medical Center, Durham, North Carolina

Supervisors: John March MD, MPH

Phoebe Moore PhD

Responsible for recruitment, conducting self reports with patients, cleaning data, data entry, facilitating cross-site collaboration, scheduling visits, preserving the blind, creating case report forms and; screening patients and controls for MRI scans.

June 2004-July 2006 Research Assistant, Treatment of Adolescent Suicide Attempters (TASA)

Department of Psychiatry, Duke University Medical Center, Durham, North Carolina

Supervisors: John March MD, MPH

Karen Wells PhD

Scott Compton PhD

Responsible for recruitment, conducting self reports with patients, cleaning data, data entry and preserving the blind

June 2004-July 2006 Research Assistant, Childhood Anxiety Multimodal Study

(CAMS)

Department of Psychiatry, Duke University Medical Center, Durham, North Carolina

Supervisors: John March MD, MPH

Scott Compton PhD

Responsible for recruitment, conducting self reports with patients, cleaning data, data entry and preserving the blind.

Aug 2003-Dec 2005 Research Assistant, Duke Coping Power Program

Department of Psychiatry, Duke University Medical Center, Durham, North Carolina

Supervisor: Karen Wells PhD

Responsibilities included conducting structured computer-based and paper assessments in subjects' homes, attrition management, conducting home-visits, scheduling assessments, collect school data and court records, consenting subjects, and supervising a team of student research assistants.

Mar 2002-Aug 2003 Undergraduate Research Assistant, Duke Coping Power Program Department of Psychiatry, Duke University Medical Center, Durham, North Carolina

Supervisor: Karen Wells 
Responsibilities included conducting structured computer-based and paper assessment measures, scheduling assessments and Service: conducting home visits.

WVU Clinical Child Training Committee

2007-2008

WVU Graduate Training Committee

2008-2009

WVU APAGS Campus Representative

2008-2009

\section{Memberships:}

Division 53 of American Psychiatric Association - Child Division Association for Behavior and Cognitive Therapies (ABCT) 